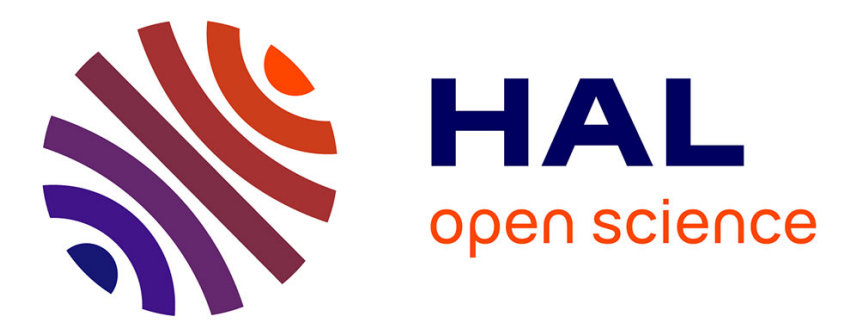

\title{
Phenothiazine-based theranostic compounds for in vivo near-infared fluorescence imaging of $\beta$-amyloid plaques and inhibition of $\mathbf{A} \beta$ aggregation
}

\author{
Yongliang Li, Jing Cai, Longjia Yan, Wanzheng Zhang, Li Li, Zhiyun Du, \\ Yanxiong Fang, Changzhi Dong, B. Meunier, Huixiong Chen
}

\section{To cite this version:}

Yongliang Li, Jing Cai, Longjia Yan, Wanzheng Zhang, Li Li, et al.. Phenothiazine-based theranostic compounds for in vivo near-infared fluorescence imaging of $\beta$-amyloid plaques and inhibition of $\mathrm{A} \beta$ aggregation. Dyes and Pigments, 2019, 171, pp.107744. 10.1016/j.dyepig.2019.107744 . hal-03108899

\section{HAL Id: hal-03108899 \\ https://hal.science/hal-03108899}

Submitted on 21 Dec 2021

HAL is a multi-disciplinary open access archive for the deposit and dissemination of scientific research documents, whether they are published or not. The documents may come from teaching and research institutions in France or abroad, or from public or private research centers.
L'archive ouverte pluridisciplinaire HAL, est destinée au dépôt et à la diffusion de documents scientifiques de niveau recherche, publiés ou non, émanant des établissements d'enseignement et de recherche français ou étrangers, des laboratoires publics ou privés.

\section{(c) (1) $\$$}

Distributed under a Creative Commons Attribution - NonCommerciall 4.0 International 


\section{List of Abbreviations:}

PCL: Poly ( $\varepsilon$ - caprolactone)

CHT: Chitosan

HUVEC: Human umbilical vein endothelial cells

EGM-2: Endothelial growth medium -2

DAPI: 6-Diamidino-2-Phenylindole

BSA: Bovine serum albumin

PBS: Phosphate-buffered saline 


\title{
Development of double porous poly $(\varepsilon$ - caprolactone $) /$ chitosan polymer as tissue engineering scaffold
}

\author{
Pritam Das ${ }^{\mathrm{a}, \mathrm{b}, \mathrm{c}}$, Jean-Christophe Remigy ${ }^{\mathrm{a}}$, Jean-François Lahitte ${ }^{\mathrm{a}}$, Andries D. van der Meer ${ }^{\mathrm{b}}$, Barbara Garmy-Susini ${ }^{\mathrm{c}}$, \\ Clémence Coetsier ${ }^{\mathrm{a}}$, Sandrine Desclauxa ${ }^{\mathrm{a}}$, Patrice Bacchin ${ }^{\mathrm{a}^{*}}$ \\ ${ }^{a}$ Laboratoire de Génie Chimique, Université de Toulouse, CNRS UMR 5503, INPT, UPS, Toulouse, France. \\ ${ }^{\mathrm{b}}$ University of Twente, Applied Stem Cell Technologies, TechMed Centre, Faculty of Science and Technology, Enschede, \\ 7500, AE, The Netherlands \\ c Institut des maladies métaboliques et cardiovasculaires (I2MC), Université de Toulouse, Inserm U1048, UPS, Toulouse, \\ France
} *Address for correspondence: Laboratoire de Génie Chimique, Université de Toulouse, CNRS, INPT, UPS, Toulouse France.
E-mail address: bacchin@ chimie.ups-tlse.fr (P. Bacchin)

\begin{abstract}
Polymer blend made from poly $(\varepsilon$ - caprolactone)/chitosan (PCL/CHT) offers interesting opportunities for biological applications. The paper presents a new way to fabricate PCL/CHT double-porosity (macrovoids with interconnected microporosity) membrane materials from a chemical optimization of the solvent and non-solvent phases and from a modified phase inversion technique. By varying the PCL/CHT proportion, it is shown that it is possible to improve the chemical and physical properties of the CHT carbohydrate polymer. The PCL/CHT membranes are fully characterized in term of physicochemical properties (ATR-FTIR, XRD and DSC) to understand the miscibility of the two-polymer blend. Morphological characterization by SEM shows that by increasing CHT wt $\%$ in the blend, the size of the macrovoids was increasing. Rapid enzymatic degradation of PCL from all the blend was found by using lipase (from P. cepacia). The mechanisms at the origin of the morphological structuration of the material is also discussed. To test the ability to operate these materials as small diameter vascular scaffolds, cell culture with human umbilical vein endothelial cells (HUVECs) were carried out on the membrane and the results analyzed with laser scanning confocal microscopy (LSCM). Data suggest that the blend membrane with higher concentration of CHT polymer wt\% have suitable properties that promote high number of cells on the surface by maintaining cellular cytoskeleton integrity within 3 days. The blend membrane with a double porous morphology could be potentially applicable in future for small diameter
\end{abstract}


vascular graft application. The surface macrovoids (20 to $90 \mu \mathrm{m})$ could be useful for three-dimensional cellular adhesion and proliferation and interconnected microporous spongy network ( 7 to $20 \mu \mathrm{m}$ ) is expected to transfer essential nutrients, oxygen, growth factor between the macrovoids and the supernatant.

Keywords: poly ( $\varepsilon$-caprolactone), chitosan, double porous, membrane, biocompatibility, cell culture

\section{Introduction}

Tissue engineering relies on the use of artificial degradable porous material scaffold integrated with biological cells or molecules to regenerate tissues [1]. The scaffold material should offer specific functionalities to accomplish this goal. Several studies show that 3D structures of the support material control the cell growth [2]. The material composition is a key point that controls the biocompatibility, the cell adhesion and optionally the biodegradability. The material engineering must be tailored to the biological strategy. In recent studies, artificial vascular grafts generally have been fabricated from synthetic polymers, such as polyester, e.g. polycaprolactone (PCL) or polylactic acid (PLA) or polylactic-co-glycolic acid (PLGA) or polyurethane $[3,4]$. This kind of polymer scaffolds can provide sufficient mechanical strength and are considered biocompatible and biodegradable without any toxic by-products. However, they are hydrophobic in nature and lack reactive functional sites for further biofunctional modification [5] which led to the development of innovative hybrid materials by mixing both synthetic and natural polymers [6]. Natural carbohydrate polymers like chitosan (CHT) on the other hand can provide an excellent candidate for biomedical vascular grafts application, mostly in combination with other synthetic polymers [7-11]. CHT is a linear polysaccharide composed of glucosamine and $\mathrm{N}$-acetyl glucosamine units connected through $\beta$-1,4-glycosidic bond and produced from the highly abundant natural polymer, chitin. It is biocompatible, bioresorbable, hydrophilic and non-toxic. It has high affinity towards proteins and provides antibacterial, hemostatic, fungistatic, antitumor and anticholesteremic properties [12-16]. It can provide a favorable environment for cell attachment as chemical structure of CHT is similar to glycosaminoglycans (GAGs), which are native components of extracellular matrix (ECM) $[17,18]$. However, it has low mechanical properties in wet 
conditions and high hydrophilicity, which can be easily optimized by blending with synthetic polymers. Blending can also improve the low degradation rate of the synthetic polymers in physiological conditions. It is well accepted that blending these two kind of polymers will be very advantageous, which can significantly reduce all the disadvantages and combine the benefits of the individual polymers providing a superior material with good tissue compatibility and sufficient mechanical strength [19-21]. The 3D structure of the material should enable to host the cells but also to distribute the nutrients and to favor the removal of cellular metabolic products.

In the last decades, various scaffolds have been developed more often from an assembly of simple fibers. The study of PCL/CHT blend with electrospinning method is extensively researched and well adaptable for better cell attachment with good dynamic properties [9,10,12,19-25]. However numerous studies report on the difficulties encountered for electrospinning pure chitosan as the structure is mechanically weak, limiting practical processing [26-29]. Furthermore, it is difficult to manage the multiple functionality requirements with an assembly of fibers. The use of membranes appears as an interesting alternative to tune the porosity and to manage the mass transport properties. The different level of membrane porosity can be adjusted in order to have both macrovoids to host the cells and interconnected micropores network to ensure the transportation of molecules [30]. Solvent mixing with PCL and CHT was more conveniently used compared to melt blending which is quite difficult to perform as CHT decomposes before undergoing melting. Although it is also difficult to make a homogeneous blend in solvent mixing due to the high viscosity of CHT in solvent and also the scarcity of good mutual solvents. Several efforts were made as Honma et al. [25,31] produced PCL/CHT blend casting by using common solvent as 1,1,1,3,3,3-hexa-fluoro-2-propanol (HFIP) solution. They showed that PCL/CHT can be processed by using HFIP but this solvent is very expensive, carcinogenic and difficult to remove during washing [32]. Although a different approach was reported to find a good mutual solvent [33-38]. Among them a suitable approach could be to dissolve CHT in $0.5 \mathrm{M}$ acetic acid and PCL in glacial acetic acid where the authors [35] were able to obtain a mixture of these two solutions at a low concentration of polymer but it was not effective at high concentration [31] as in our case. In another study, a solvent mixture of formic acid/acetone was reported to be used to develop PCL/CHT nanofibers at high 
concentration of polymers. In this case, it was forming beads-like morphology indicating incomplete dissolution of the polymer [39]. Recently, formic acid/acetic acid mixture [40] with certain ratio has produced a mutual solvent for both polymers with minimal degradation and phase segregation but with faster dissolution. Despite so many combination of solvents, the author [40] have reported that the formic acid/acetic acid mixture produced the bead free and least toxic scaffold and the solvent can be easily removed during washing.

Here we propose a new scaffold based on polymer membrane technologies to meet the needs and the constraints discussed above. The main objectives are to obtain a biocompatible and biodegradable material with open macrovoids for three-dimensional cell culture and interconnected microporosities to transport nutrients, oxygen, between the macrovoids and supernatant. The membranes were fabricated from a PCL/CHT blend with a modified liquid induced phase inversion technique in order to create macrovoids on the surface. The percentage ratio of PCL/CHT (100/0, 90/10, 80/20, 70/30 w/w \%) blend was changed to investigate a range of chemical interactions, miscibility and biodegradability. The morphology of the developed materials was observed by SEM and the physico-chemical properties of the blends were characterized by ATR-FTIR mapping, XRD and DSC. In addition, enzymatic degradation study was performed by using lipase (from Pseudomonas cepacia; $7 \mathrm{U} / \mathrm{ml}$ ) at $37{ }^{\circ} \mathrm{C}$ in phosphate buffer saline (PBS) at physiological pH. Change in morphological properties and weight loss (\%) were monitored at predetermined time intervals of the degradation study. To test the biocompatibility and potentialities of the membranes as a small diameter vascular graft for cardiovascular system disease, cell culture was performed with human umbilical vein endothelial cells (HUVECs) up to 3 days and results were observed in laser scanning confocal microscopy.

\section{Materials and methods}

\subsection{Materials}

CHT (MW 190-310 kDa, 75-85\% de-acetylation degree, CAS Number 9012-76-4) was purchased from Sigma-Aldrich and PCL (MW 80 kDa, CAPA ${ }^{\mathrm{TM}}$ 6800, CAS Number 24980-41-4) was purchased from Perstorp Holding AB, Sweden. Isopore ${ }^{\circledR}$ Polyester (PET) track-etched membranes (with $10 \mu \mathrm{m}$ pore diameter), used as macrovoid generator, were purchased from Sterlitech, USA. Acetic acid, formic acid, 
sodium hydroxide and sodium azide $\left(\mathrm{NaN}_{3}\right)$ were purchased from Sigma-Aldrich. Lipase from Pseudomonas cepacia (activity: 35U/mg, CAS Number 9001-62-1) were purchased from SigmaAldrich. Phosphate buffer saline (PBS) was purchased from Fisher Scientific (CAS Number 7778-770). Human umbilical vein endothelial cells (HUVECs) were purchased from Lonza and the corresponding culture medium (EGM-2: basal medium with supplement mix, CC-22011) was obtained from Promocell. Collagen I (rat-tail) were obtained from Santa Cruz. Trypsin-EDTA, Formaldehyde, 6Diamidino-2-Phenylindole (DAPI), and Alexa Fluor 488 Phalloidin were purchased from Thermo Fisher. Triton X-100, bovine serum albumin (BSA) were purchased from Sigma Aldrich.

\subsection{Methods}

\subsubsection{Development of the membrane with a double porosity level}

The membranes were developed by modified liquid induced phase inversion method by diffusion between a solvent as formic acid/acetic acid (FA/AA) mixture and non-solvent as $\mathrm{NaOH}$ aqueous solution. PCL and CHT with a ratio 100/0, 90/10, 80/20 and 70/30 (w/w\%) were dissolved in FA/AA $(\mathrm{w} / \mathrm{w} \%)$ mixture. The polymer concentration in the solution was expressed in wt.\% as followed:

CHT (wt. \%) $=100 \frac{\text { Mass }_{\mathrm{CHT}}}{\text { Mass }_{\mathrm{CHT}}+\operatorname{Mass}_{\mathrm{PCL}}}$

Polymer (wt. \%) $=100 \frac{\text { Mass }_{\mathrm{CHT}}+\text { Mass }_{\mathrm{PCL}}}{\operatorname{Mass}_{\mathrm{CHT}}+\text { Mass }_{\mathrm{PCL}}+\text { Mass }_{\text {Solvents }}}$

The polymer solution was casted on a glass plate using a casting knife in order to achieve a thickness around $250 \mu \mathrm{m}$ at $23{ }^{\circ} \mathrm{C}$. The track-etched membrane was at first immersed in the solvent mixture to fill the pores with solvent mixture, slowly wiped out to remove excess solvent and slowly applied on the casted polymer solution (Fig. 1). 


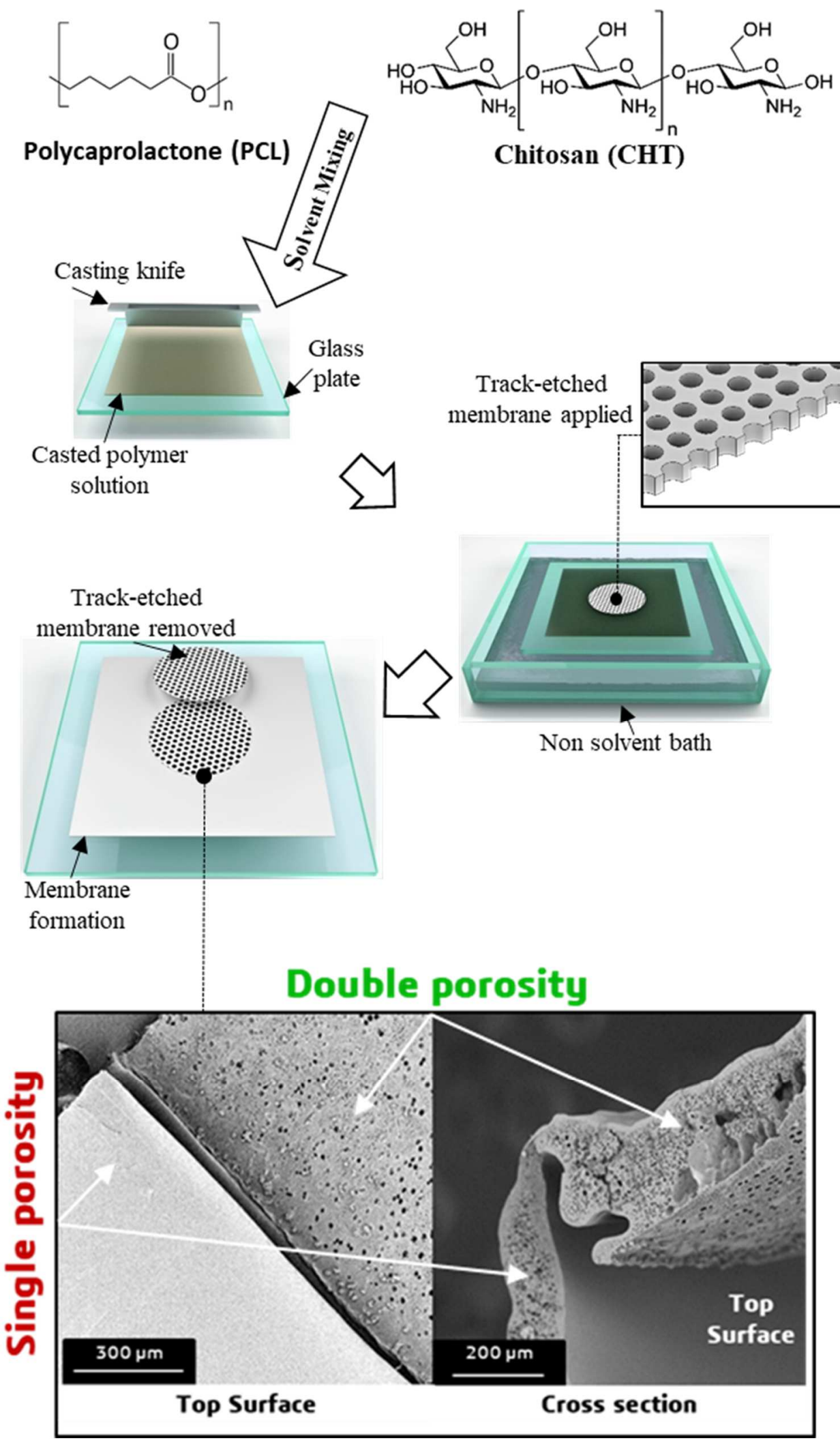

Fig. 1. Double porosity development by modified liquid induced phase inversion process with the following steps: PCL/CHT membrane casting, applying the track-etched membrane before immersing in non-solvent bath and removing track-etched membrane after membrane formation. SEM images of PCL/CHT 100/0 represent double porosity and single porosity on top surface and cross section of the same membrane. 
The glass plate was then gently immersed in a non-solvent bath containing $\mathrm{NaOH}$ solution at $23{ }^{\circ} \mathrm{C}$. Immediately after the immersion, demixing via neutralization of the acidic casted solution was started due to solvent exchange leading to the flat sheet membrane formation in few minutes (in case of PCL/CHT 100/0) to hours (in case of PCL/CHT 70/30). It should be noted that this quenched phase inversion method leads to a thermodynamically non-equilibrium state of the polymers in the final membrane and two different kinds of porous structures were formed in the same condition Fig. 1.

The solvent exchange rate was spatially quite homogeneous where the polymer solution was not covered by the track-etched membrane leading to single porous structure without enough open pores on the top surface. On the other hand, where the polymer solution was covered by the track-etched membrane, a heterogeneous solvent exchange was occurred leading to the formation of double porous structure. The macrovoids were formed where the non-solvent has a direct access to the polymer solution through the pores of the track-etched membrane. And, interconnected micropores were formed by diffusion of the non-solvent through the newly formed macrovoids. This mechanism was described by Strathmann et al. [41] to explain the formation of skinned membrane with macrovoids and sponge structure; the nascent skin playing an equivalent role like our track-etched membrane as discussed in Sec. 3.7.

After the membrane formation, when it turned opaque and detached from the glass plate, the tracketched membrane was removed gently. The PCL/CHT membrane was washed several times in ultrapure water and stored in ultrapure water at $4{ }^{\circ} \mathrm{C}$.

The surface where the macrovoids were open towards the non-solvent (i.e. where the track-etched membrane was applied) is denoted as top surface and the other surface, which was facing the glass plate, is denoted as the bottom surface.

\subsubsection{Viscosity measurements of the polymer solution}

The viscosity of the polymer solution was measured by Rheometer Anton Paar Physica MCR 301, France. After complete dissolution of the solution, a $0.6 \mathrm{ml}$ volume was injected on a cone plate rheometer (diameter $50 \mathrm{~mm}$ ). The viscosity was measured at a shear rate from 2 to $100 \mathrm{~s}^{-1}$ at $20^{\circ} \mathrm{C}$. All 
the measurements were performed inside a close chamber in order to avoid the solvent evaporation and dissolution of water from air moisture.

\subsubsection{Scanning electron microscopic (SEM) analysis}

SEM analysis (Phenom XL, Fondis Biotech, France) were done on both double and single porous membranes to evaluate all the developed blend morphology from the top surfaces and the cross sections. All the samples were coated with gold and analysis were performed at an accelerating voltage of $10 \mathrm{kV}$. The surface porosity was also determined from the SEM images by using ImageJ [42] software and reported in Table 1.

\subsubsection{Attenuated total reflection (ATR)-Fourier transformed infrared (FTIR) spectroscopic analysis and mapping}

FTIR analysis were done by using a Nexus Nicolet (USA) FTIR Microscope system with an ATR diamond crystal at $45^{\circ}$ angle. Prior to the examination, $1 \mathrm{~cm}^{2}$ sample was cut and measurements were done on both surfaces (three times) of each sample. Each point was scanned sixteen times with a resolution of $8 \mathrm{~cm}^{-1}$ and the spectral range was $650-4000 \mathrm{~cm}^{-1}$. The ATR correction was applied on all the spectra [43]. FTIR chemical mapping on either side of the membrane also performed using an infrared spectrometer IN10MX Thermoscientific (USA) with an ATR germanium crystal of a $25^{\circ}$ angle. A square sample of $6 \mathrm{~mm}^{2}$ was studied. Data were analyzed on a $50 \mu \mathrm{m} \times 50 \mu \mathrm{m}$ surface for each point (one point was measured every $50 \mu \mathrm{m}$ ) with a spectral resolution of $8 \mathrm{~cm}^{-1}$. Each measurement was scanned by sixteen times with the spectral range $650-4000 \mathrm{~cm}^{-1}$.

\subsubsection{X-ray diffraction (XRD) analysis}

The crystalline structure of membrane sample $(5 \mathrm{~mm} \times 5 \mathrm{~mm})$ was investigated by XRD analysis using a D4 Endeavor X-ray diffractometer $\left(\mathrm{CuK} \alpha_{1}=0.154056 \mathrm{~nm}\right.$ and $\mathrm{CuK} \alpha_{2}=0.154044 \mathrm{~nm}$; generator 40 $\mathrm{eV}$; $40 \mathrm{~mA}$, Bruker AXS, Karlsruhe, Germany) from $10^{\circ}$ to $100^{\circ}$ at a scan speed of $21.7 \mathrm{sec} / \mathrm{step}$ that is equal to $0.02^{\circ}$ (2-theta).

\subsubsection{Differential scanning calorimetric (DSC) measurements}

DSC experiments were conducted (DSC TA instrument Q2000, France) in order to evaluate thermal properties of the PCL/CHT blend membranes. The membranes were dried at $37^{\circ} \mathrm{C}$ and were cut in a 
dimension of $3 \mathrm{~mm}^{2}$. The sample was put inside an aluminum pan and mechanically covered by an aluminum cap. The pre-weighted aluminum pan was placed inside the DSC machine and equilibrate at $20{ }^{\circ} \mathrm{C}$. The temperature scanning was performed at a constant heating rate of $10{ }^{\circ} \mathrm{C} \mathrm{min}^{-1}$, from 20 to 80 ${ }^{\circ} \mathrm{C}$ without preheating step. The melting point $\left(\mathrm{T}_{\mathrm{m}}\right)$ of PCL in the blend by varying $\mathrm{CHT}$ wt.\% (pure PCL melting point is $60^{\circ} \mathrm{C}$ ) determined.

\subsubsection{Enzymatic degradation of the blend}

The membranes were dried in vacuum at $37^{\circ} \mathrm{C}$ until constant weight was reached and were cut in $6 \mathrm{~mm}$ $\times 6 \mathrm{~mm}$ pieces. The initial weight $\left(\mathrm{w}_{\mathrm{i}}\right)$ was taken (in mg up to the fifth decimal point). Then the samples were placed in a $5 \mathrm{ml}$ vials containing $3 \mathrm{ml}$ of PBS $1 \times$ solution, $0.6 \mathrm{mg}(7 \mathrm{U} / \mathrm{ml})$ of lipase (from Pseudomonas cepacia) and 0.05 wt. $\% \mathrm{NaN}_{3}$. The entire procedure was done in a sterile condition and vials were sealed and stored in incubator at $37^{\circ} \mathrm{C}$. The enzyme solution was changed every day in order to maintain the same enzymatic activity during the whole process. The same procedure was done without the enzyme as a control. Three consecutive samples were taken out after 6 hours of incubation and every one-day intervals until 10 days. The samples were washed with copious deionized water, dried at $37^{\circ} \mathrm{C}$ in vacuum until constant weight. The final weight, $W_{f}$, was taken.

The weight loss, $W_{\text {loss }} \%$, was calculated by the following equation:

$W_{\text {loss }} \%=100 \frac{W_{i}-W_{f}}{W_{i}}$

Where, $W_{i}$, is the initial weight and, $W_{f}$, the final weight.

Scanning electron microscopic images of the degraded membranes were taken and the ageing solution was collected to observe the change in $\mathrm{pH}$.

\subsubsection{Cell culture and study by laser scanning confocal microscopy}

Membranes were cut with diameter of $2.2 \mathrm{~cm}$ and washed with sterile phosphate-buffered saline (PBS) three times. The samples were put in 12 well plate and kept in sterile PBS suspension at $4{ }^{\circ} \mathrm{C}$ for overnight before the cell seeding. Then the samples were washed again with sterile PBS, $1 \mathrm{ml}$ collagen I $(0.1 \mathrm{mg} / \mathrm{ml}$ in PBS $)$ was added in the well plate and incubated for 30 minutes at $37^{\circ} \mathrm{C}$. Then the well 
plate was taken out from the incubator, washed with PBS and incubated for 1 hour with EGM-2 culture medium (Lonza). Meanwhile, human umbilical vein endothelial cells (HUVECs from Lonza) were trypsinized (passage 4) and collected from a confluent T175 flask. The well plate was taken inside a sterile hood and the incubated culture medium was discarded. Then, $3 \mathrm{ml}$ of homogeneous cell suspension $\left(4 \times 10^{6}\right.$ cells $/ \mathrm{ml}$ or $10^{6}$ cells $\left./ \mathrm{cm}^{2}\right)$ was pipetted in each well plate and incubated at $37{ }^{\circ} \mathrm{C}$ for 2 hours to ensure complete attachments. The well plate was taken out of the incubator, replaced with fresh culture medium to remove non-attached cells and incubated again.

After 3 days, the well plates were taken in sterile hood and prepared for cell staining. At first the well plates were washed with PBS and the cells were fixed by adding $4 \%$ formaldehyde for 15 minutes. Then the wells were washed with PBS and kept in a permeabilization buffer (PB; 9:1 v:v ratio of $11 \mathrm{mg} / \mathrm{ml}$ BSA with $1 \%$ Triton $\mathrm{X}-100$ in PBS) at room temperature for 15 minutes to block any non-specific protein-binding sites. Phallodin-488 and DAPI (Actin Green 488, NucBlue, Thermo Fisher Scientific) were added together in the concentration of $1 \mathrm{drop} / \mathrm{ml}$ and 1:4000 in PB, respectively, and incubated at room temperature for 1 hour. To prevent photo-bleaching, the well plates were protected from light exposure from this step onwards. Then the well plates were washed thoroughly with PBS and imaged with confocal microscopy (Zeiss LSM 510, Nikon) in 20x and 60x magnification (Fig. 12.). As supplementary information, other biological characterizations have been performed with human mesenchymal stem cells (MSCs) in a successive z-stack confocal analysis on these 3D scaffolds [44] and the hydraulic permeability of HUVECs layers have been measured in a microfluidic organ-on-chip device $[44,45]$.

\section{Results and discussion}

\subsection{Polymer-solvent-non solvent optimization}

Chitosan (CHT) and polycaprolactone (PCL) are polymers with very different chemical properties and finding a common solvent to develop a membrane was an important challenge. Moreover, maintaining an optimum viscosity was very important to achieve the double porous membrane structure. The solvent optimization have been partially based on viscosity measurement of the polymer casted solution (Fig. 2) as it was an important parameter that directed the size of the macrovoids during solvent exchange. 
During solvent mixing, a visible reduction of the viscosity has been observed by dissolving PCL in the common solvent after $2-3$ hours at temperature more than $35^{\circ} \mathrm{C}$, probably due to the hydrolysis of ester bonds of PCL. In order to avoid the reduction of viscosity, different factors were tuned. CHT was dissolved in the solvent mixture at first at $55^{\circ} \mathrm{C}$ for $12 \mathrm{~h}$ and then PCL was added when the temperature was below $35^{\circ} \mathrm{C}$. In the common solvent, formic acid was found more responsible for the breakage of PCL ester bonds, although higher wt $\%$ of formic acid was better for faster and bead free dissolution. For these reasons, the solvent was also optimized for each PCL/CHT polymer blend according the following composition: PCL/CHT 100/0 (15 wt.\%) in FA/AA 70/30 (w/w) \%, PCL/CHT 90/10 and 80/20 (14 wt. \%) in FA/AA 60/40 (w/w) \% and PCL/CHT 70/30 (10 wt\%) in FA/AA 50/50 (w/w) \% (Table 1). In these conditions of composition and temperature, a clear, light yellow and viscous solution was obtained within two hours. After this dissolution step, the polymer solution was kept in stand for 10-20 minutes before starting the membrane casting. Due to high viscosity of CHT in the solvent mixture, PCL/CHT blend beyond 70/30 ratio was not successful as it was difficult to make a homogeneous mixture of polymer solution by mechanical stirrer and to avoid phase segregation between the two polymers. Viscosity of CHT was remarkably high in the solvent mixture (Fig. 2) that dissolving pure CHT above $4 \mathrm{wt} . \%$ was difficult and concentration lower than $4 \mathrm{wt} . \%$ are not sufficient to make a double porous membrane certainly due to lack of good entanglement between the polymer chain or a phase inversion leading to polymer particles.

The viscosity of the final polymer solution of pure PCL, CHT and the different blends were measured and reported in Fig. 2. Results showed that the viscosity of pure CHT of only 4 wt.\% solution was significantly higher than the viscosity of PCL/CHT 100/0 of $15 \mathrm{wt} . \%$ solution in the common solvent. This was due to several factors. First of all, the molecular weight of CHT (190 kDa - $310 \mathrm{kDa})$ was higher than the molecular weight of PCL $(80 \mathrm{kDa})$. Secondly, CHT has higher affinity towards the solvent due to the presence of stronger secondary interaction between the functional groups of CHT and the common solvent. As a result, CHT polymer chains likely to be deployed in the solvent leading to higher viscosity [46]. On contrary, in acidic solvent, the viscosity of PCL could be decreased to some 
extent due to a decrease of the polymer chain length via breakdown of ester linkages. All these effects are responsible for the high difference of solution viscosity between these two polymers.

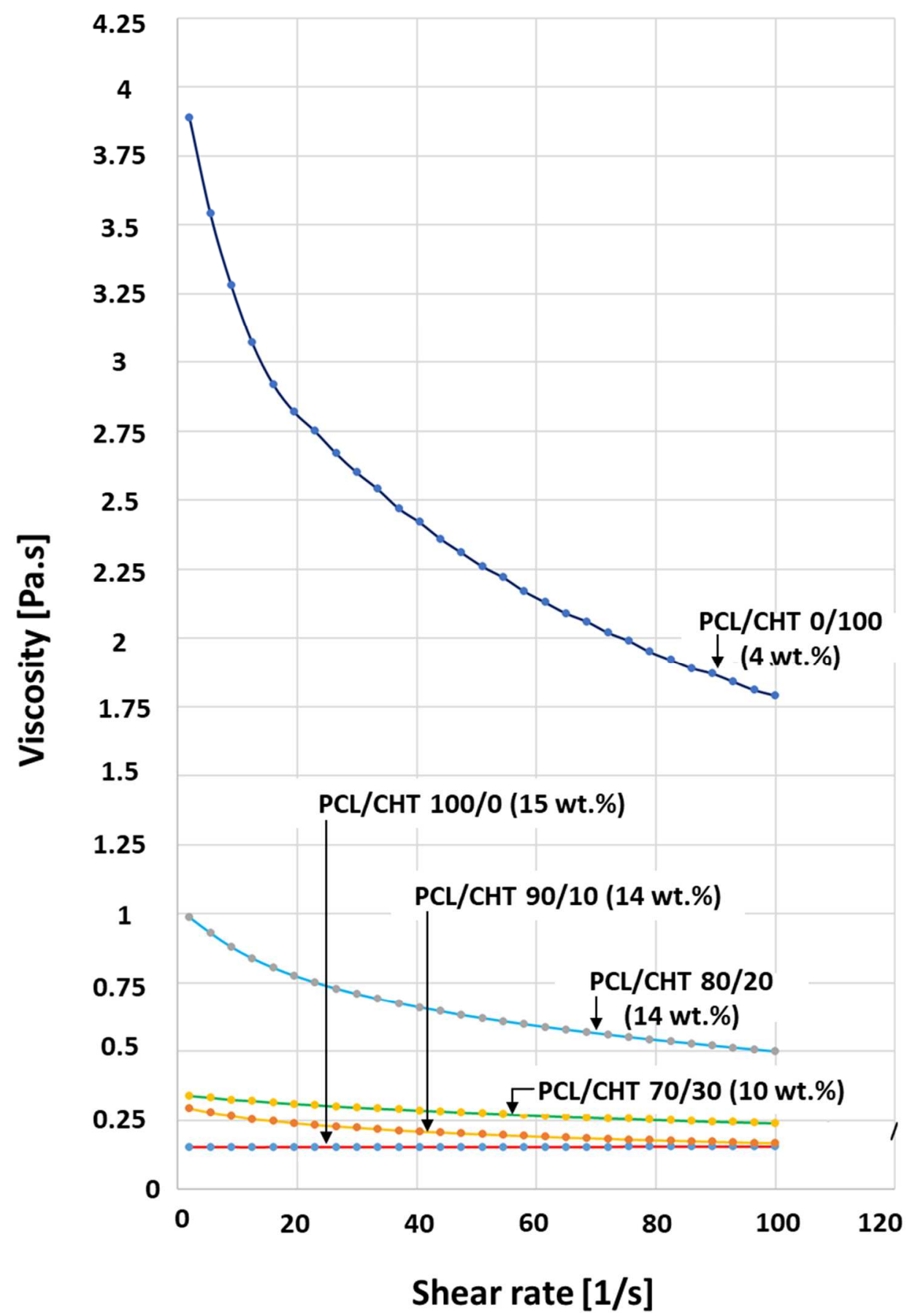

Fig. 2. Viscosity [Pa.s] vs shear rate [1/s] measurement of PCL/CHT 100/0 (15 wt.\%), PCL/CHT 90/10 (14 wt.\%), PCL/CHT 80/20 (14 wt.\%), PCL/CHT 70/30 (10 wt.\%) and PCL/CHT 0/100 (4 wt.\%) scaffolds respectively in solvent FA/AA mixture. 
Concerning the non-solvent, concentration of $\mathrm{NaOH}$ more than $0.5 \mathrm{M}$ in the non-solvent bath was responsible to make the membrane fragile by breaking the ester bonds in PCL during membrane formation. Concentration lower than $0.2 \mathrm{M}$ was not sufficiently concentrated to allow the formation of macrovoids. We also varied the concentration of the $\mathrm{NaOH}$ solution depending upon the viscosity of the casted solution in order to get consistent macrovoids height of about $50-70 \%$ of the total membrane thickness. Finally, a 0.3 M NaOH solution was used for PCL/CHT 100/0 (15 wt.\%) and 0.5 M NaOH solution was used for the rest of the PCL/CHT blend.

\subsection{Morphology of the double porous membrane}

After the membrane fabrication, the top surface and the cross section morphological characteristics were observed in SEM (Fig. 3.). All the scaffolds, which were produced by using the track-etched membrane, are showing double porosity where the macrovoids are open towards the top surface and connected with the spongy microporous network. From the cross section view, it was observed that the localized arrival of the non-solvent inside the polymer solution were creating the macrovoids which were increasing by increasing CHT wt.\%. The macrovoids diameter were around $20 \pm 3-90 \pm 5 \mu \mathrm{m}$ and the diameter of interconnected micropores were around $7 \pm 3 \mu \mathrm{m}$ (except the PCL/CHT 70/30 ratio for which the micropore diameter was $20 \pm 5 \mu \mathrm{m}$ (Table 1)). 

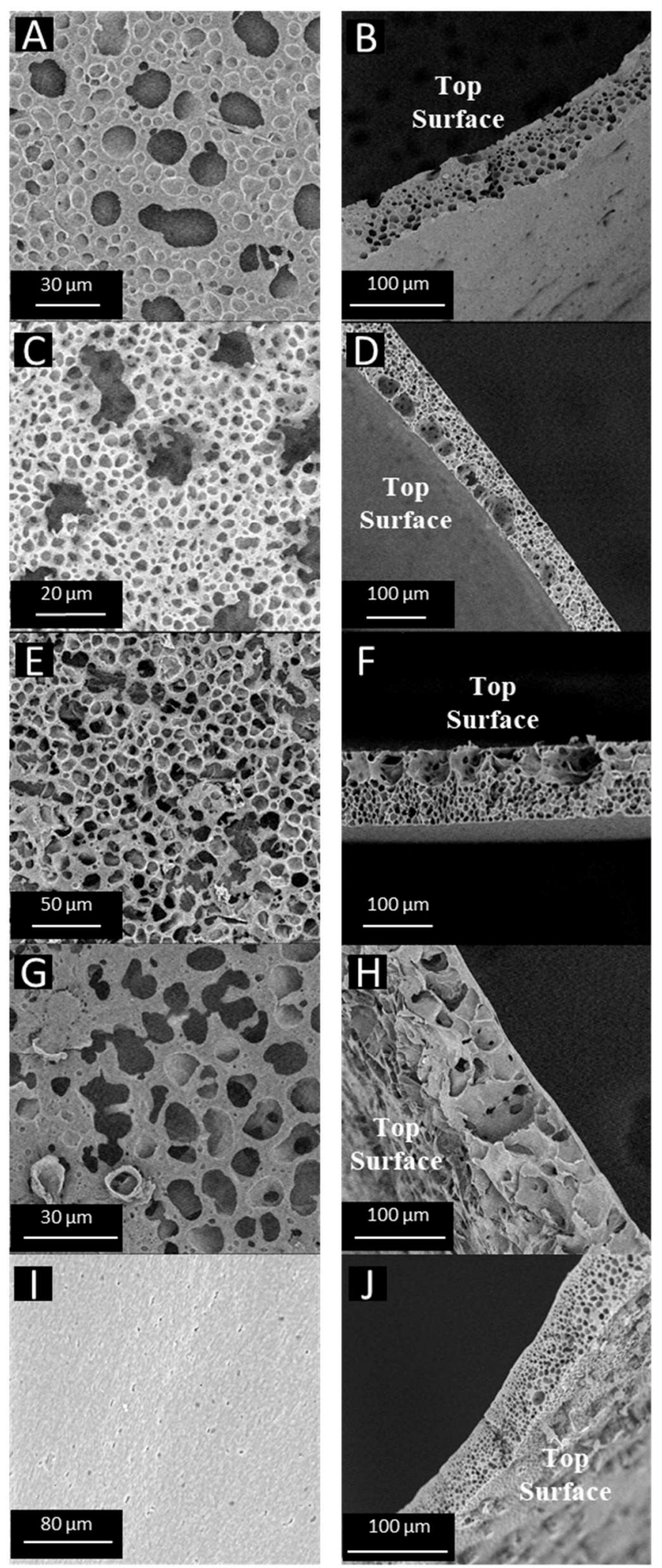

Fig. 3. SEM micrographs of membranes on the top surface and cross section with double porosity (using track-etched membrane): (A, B) PCL/CHT 100/0, (C, D) PCL/CHT 90/10, (E, F) PCL/CHT 80/20, (G, H) PCL/CHT 70/30. Single porosity (without using track-etched membrane): (I, J) PCL/CHT 100/0, top surface and cross-section, respectively. 
Table 1. Summary of the polymer blend membrane properties with distinct concentration in correlation with viscosity and pore diameter, melting point.

\begin{tabular}{|c|c|c|c|c|c|}
\hline & \multicolumn{5}{|c|}{ PCL/CHT percentage Ratio } \\
\hline & $100 / 0$ & $90 / 10$ & $80 / 20$ & $70 / 30$ & $0 / 100$ \\
\hline $\begin{array}{l}\text { Total Polymer } \\
\text { Conc. }(w / w \%)\end{array}$ & 15 & 14 & 14 & 10 & 4 \\
\hline $\begin{array}{c}\text { Solvents } \\
\mathrm{HCOOH} / \mathrm{AcOH} \\
(\mathrm{w} / \mathrm{w} \%)\end{array}$ & $70 / 30$ & $60 / 40$ & $60 / 40$ & $50 / 50$ & $50 / 50$ \\
\hline $\begin{array}{l}\text { Nonsolvent } \\
\mathrm{NaOH}(\mathrm{M})\end{array}$ & 0.3 & 0.5 & 0.5 & 0.5 & - \\
\hline $\begin{array}{c}\text { Macrovoid } \\
\text { diameter }(\mu \mathrm{m})^{*}\end{array}$ & $20 \pm 3$ & $56 \pm 5$ & $67 \pm 5$ & $90 \pm 5$ & - \\
\hline $\begin{array}{c}\text { Micropore } \\
\text { diameter }(\mu \mathrm{m})^{*}\end{array}$ & $7 \pm 3$ & $7 \pm 3$ & $7 \pm 3$ & $20 \pm 5$ & - \\
\hline $\begin{array}{c}\text { Surface } \\
\text { Porosity }(\%)^{*}\end{array}$ & $40 \pm 5$ & $50 \pm 5$ & $53 \pm 5$ & $45 \pm 5$ & - \\
\hline $\begin{array}{l}\text { Viscosity of } \\
\text { polymer sol. } \\
\text { (Pa.s)@ shear } \\
\text { rate } 2 \mathrm{sec}^{1 \S}\end{array}$ & 0.153 & 0.293 & 0.987 & 0.338 & 3.89 \\
\hline $\begin{array}{l}\text { M.P. of PCL in } \\
\text { the Blend (Tm) } \\
{ }^{\circ} C^{\Psi}\end{array}$ & 61.59 & 62.78 & 63.19 & 61.70 & - \\
\hline
\end{tabular}

\subsection{Chemical characterization by ATR-FTIR spectra}

The representative FTIR spectra of the bottom surface of the membrane are shown in Fig. 4. (A). All the stretching and bending vibrations are found to be well matching with the reported values [31]. In crude CHT, the broad peak at $3364 \mathrm{~cm}^{-1}$ was found due to stretching vibration of intramolecular hydrogen bonding between $\mathrm{N}-\mathrm{H}$ and $\mathrm{O}-\mathrm{H}$. The peak at $2880 \mathrm{~cm}^{-1}$ was due to the asymmetric bending of C-H group. The N-H and C-O-C peaks were observed at $1651 \mathrm{~cm}^{-1}$ and $1070 \mathrm{~cm}^{-1}$ respectively. In PCL, the peaks at $2946 \mathrm{~cm}^{-1}$ and $1726 \mathrm{~cm}^{-1}$ represent the characteristic peaks for $\mathrm{C}-\mathrm{H}$ and ester carbonyl $(\mathrm{C}=\mathrm{O})$ groups respectively. In PCL/CHT blend, by decreasing CHT proportion, the stretching vibration of intramolecular hydrogen bonding between $\mathrm{N}-\mathrm{H}$ and $\mathrm{O}-\mathrm{H}$, shifted towards higher wavenumber region from $3364 \mathrm{~cm}^{-1}$ in PCL/CHT 70/30 to $3437 \mathrm{~cm}^{-1}$ in PCL/CHT 90/10. In addition, the C-H stretching of PCL shifted also towards higher wavenumber region from $2943 \mathrm{~cm}^{-1}$ in PCL/CHT 70/30 to $2946 \mathrm{~cm}^{-1}$ 
in PCL/CHT 90/10. From all these results, no additional peak was observed indicating presence of no covalent bonding between the two polymers occurred. However, it is clear that the characteristic peak of the blend membrane were constantly shifting by changing the composition likely due to the secondary interaction between the functional groups of two polymers [20,47]. These interactions indicate no phase separation occurs between CHT and PCL in accordance with Sarasam et al. [34] and She et al. [38] who observed a negative Flory Huggins parameter for these two polymers.

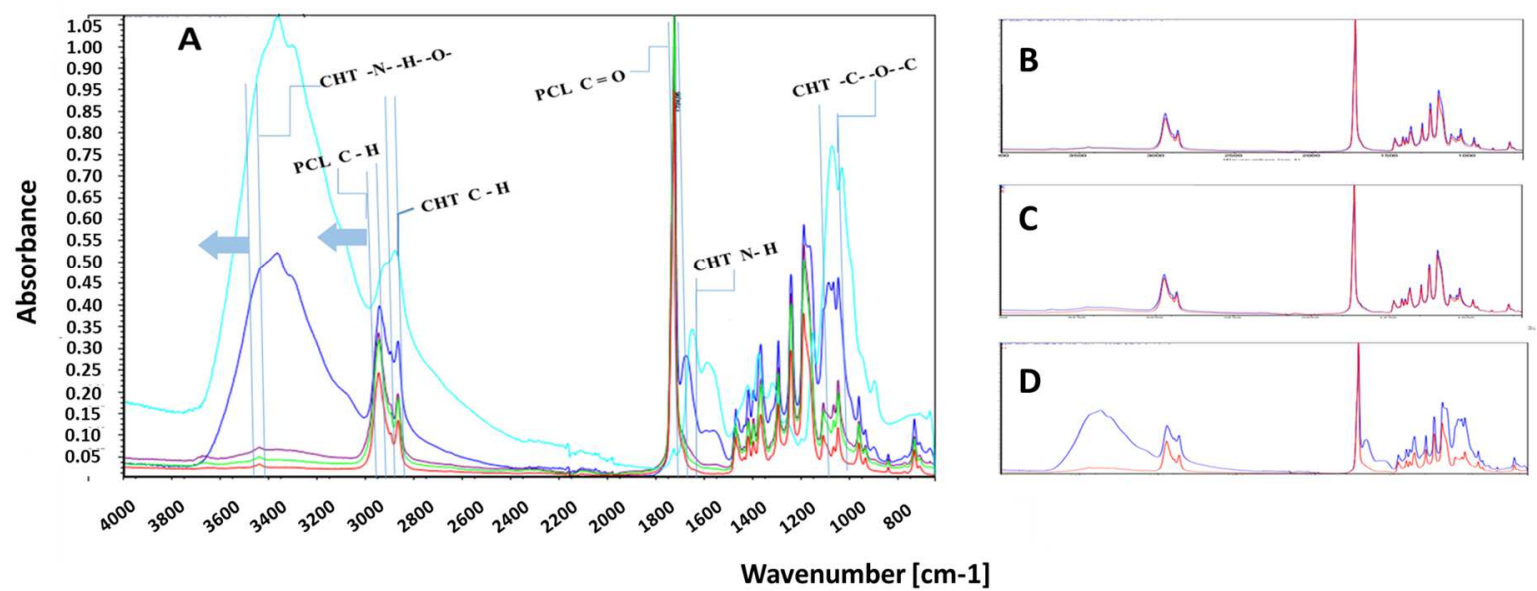

Fig. 4. (A) ATR-FTIR spectra of PCL/CHT scaffold on bottom surface where; PCL/CHT 100/0 in red, PCL/CHT 90/10 in green, PCL/CHT 80/20 in purple, PCL/CHT 70/30 in blue and CHT crude in cyan color respectively. (B, C \& D) ATR-FTIR spectra of PCL/CHT 90/10, 80/20 \& 70/30 scaffold respectively on either side of same membrane where; top surface in red and bottom surface in blue color respectively.

The penetration depth of ATR-FTIR absorption, $d_{p}$, for a sample is given by the following equation [31]:

$d_{p}=\frac{\lambda}{2 \pi \sqrt{\sin ^{2} \theta-n_{21}}}$

where, $\lambda$ and $\theta$ are the wavelength and incident angle $\left(30^{\circ}\right)$ respectively. $n_{21}$ is the ratio of refractive index of the sample to that of prism. The refractive indices of germanium and polymeric materials are 4.0 and $\approx 1.5,[31]$ respectively. By putting the wavenumbers corresponding to the characteristic absorption, we obtain a penetration depth around $\approx 3 \mu \mathrm{m}$, which means that the ATR-FTIR spectra could 
only gather the information from first few micrometer depth of the surface, whereas the thickness of the membrane sample were around $80 \mu \mathrm{m}$.

The comparison of ATR-FTIR spectra for either side of the same blend membrane is presented in Fig. $4(\mathbf{B}, \mathbf{C}, \mathbf{D})$. One can note that the intensities of specific bands of PCL and CHT are almost the same on the both surface of PCL/CHT 90/10 and PCL/CHT 80/20 membrane indicating almost homogeneous blend. Whereas, in the case of PCL/CHT 70/30, the CHT characteristic peak were higher on the bottom surface indicating probable phase segregation of PCL and CHT through the membrane thickness. From this ATR-FTIR study, one can conclude no phase separation occurs due to the interaction between PCL and CHT but a segregation could occur through the membrane thickness from the composition of PCL/CHT 70/30 due to distinguished chemical properties of the polymer mentioned earlier.

ATR-FTIR chemical mapping (Fig. 5) of two characteristic bands were observed to highlight the difference of the membrane surface chemistry; $\mathrm{C}=\mathrm{O}\left(1726 \mathrm{~cm}^{-1}\right)$ for PCL and intramolecular hydrogen bonded $\mathrm{N}-\mathrm{H}$ and $\mathrm{O}-\mathrm{H}\left(3364 \mathrm{~cm}^{-1}\right)$ for CHT [21]. 
Phenothiazine-based theranostic compounds for in vivo near-infared fluorescence imaging of $\beta$-amyloid plaques and inhibition of $\mathrm{Ab}$ aggregation

YongLiang Li, Jing Cai, Longjia Yan, Wanzheng Zhang, Le Li, Zhiyun Du, Yan XiongFang, ChangZhi Dong, Bernard Meunier, Huixiong Chen

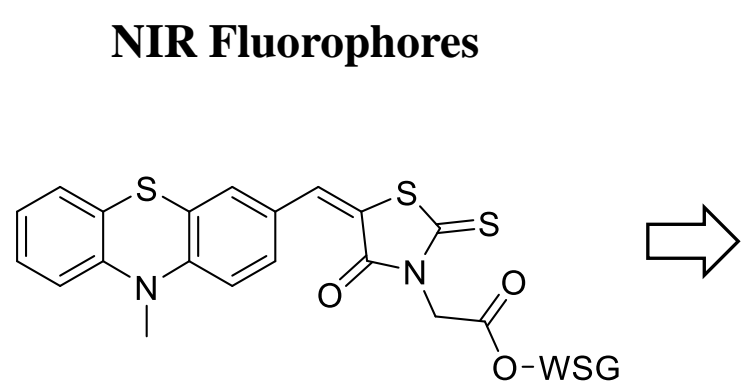

WSG $=$ Water Solubilizing Group

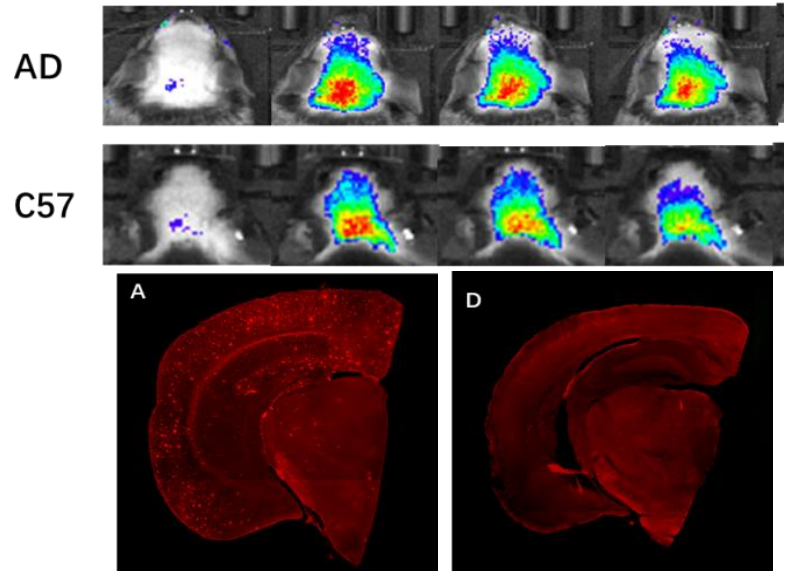

